\title{
Experimental validation of a numerical 3-D finite model applied to wind turbines design under vibration constraints: TREVISE platform
}

\author{
Takwa Sellami ${ }^{1,2,}$, Sana Jelassi ${ }^{1}$, Abdel Moumen Darcherif ${ }^{1}$, Hanen Berriri ${ }^{2}$, and Med Faouzi Mimouni ${ }^{2}$ \\ ${ }^{1}$ ECAM-EPMI and Quartz-Lab, EA 9373, 95000 Cergy-Pontoise Cedex, France \\ 2 ENIM, LASEE, 5000 Monastir Cedex, Tunisie
}

Received: 12 December 2016 / Accepted: 1 December 2017

\begin{abstract}
With the advancement of wind turbines towards complex structures, the requirement of trusty structural models has become more apparent. Hence, the vibration characteristics of the wind turbine components, like the blades and the tower, have to be extracted under vibration constraints. Although extracting the modal properties of blades is a simple task, calculating precise modal data for the whole wind turbine coupled to its tower/foundation is still a perplexing task. In this framework, this paper focuses on the investigation of the structural modeling approach of modern commercial micro-turbines. Thus, the structural model a complex designed wind turbine, which is Rutland 504, is established based on both experimental and numerical methods. A three-dimensional (3-D) numerical model of the structure was set up based on the finite volume method (FVM) using the academic finite element analysis software ANSYS. To validate the created model, experimental vibration tests were carried out using the vibration test system of TREVISE platform at ECAM-EPMI. The tests were based on the experimental modal analysis (EMA) technique, which is one of the most efficient techniques for identifying structures parameters. Indeed, the poles and residues of the frequency response functions (FRF), between input and output spectra, were calculated to extract the mode shapes and the natural frequencies of the structure. Based on the obtained modal parameters, the numerical designed model was up-dated.
\end{abstract}

Keywords: Wind turbine-tower-foundation / finite volumes method / vibration measure / experimental modal analysis / impact hammer / shaker / TREVISE platform

\section{Introduction}

Wind energy is still one of the first effective solutions for environmental degradation conditions, energy crisis and global warming. The wind energy capacities are expected to carry on being the essential source of energy around the world. The Global Wind Energy Council (GWEC) declares that more than $35 \mathrm{GW}$ of new wind power capacity was brought online in 2013 [1]. The installed capacity of the global wind farm has reached now around $486.8 \mathrm{GW}$. At the end of 2021, the GWEC estimates that the total installed capacity in the world is expected to reach $817 \mathrm{GW}$ [2].

The windiest areas of countries are still capturing researchers to install hybrid renewable energy systems operating in both grids connected and standalone modes. Tunisia is one of countries where wind and solar radiations are two abundantly available energy resources especially in the northeast of the Tunisian territory with wind speed

\footnotetext{
* e-mail: sellami.takwa8@gmail.com
}

levels of $10-15 \mathrm{~m} / \mathrm{s}$ as shown in Figure 1. The wind power capacity could attain $1 \mathrm{GW}$ if the clean energy potentials were fully exploited [3]. Unfortunately, only $11.4 \%$ of it is explored in 2013. Thus, the country aims to realize new projects in order to produce $1 \mathrm{GW}$ of electricity from both wind and photovoltaic resources by the end of 2020 and $4.7 \mathrm{GW}$ by the end of 2030 [4].

Given the complexity of micro-turbine systems combining electrical, mechanical and structural processes, the instability of the structure is often expected [5]. Accordingly, a structural model of the used wind turbine system has to be successfully set up. The natural frequencies, mode shapes and damping, called the modal parameters, represent the characteristics of the structural model [6,7].

Decades ago, the fast advance of numerical tools complemented by the notable development in personal computers technologies drove designers to take an interest to software packages-based techniques for parameter identification of structures. Thus, a major attention was assigned to the progress of robust finite elements and 


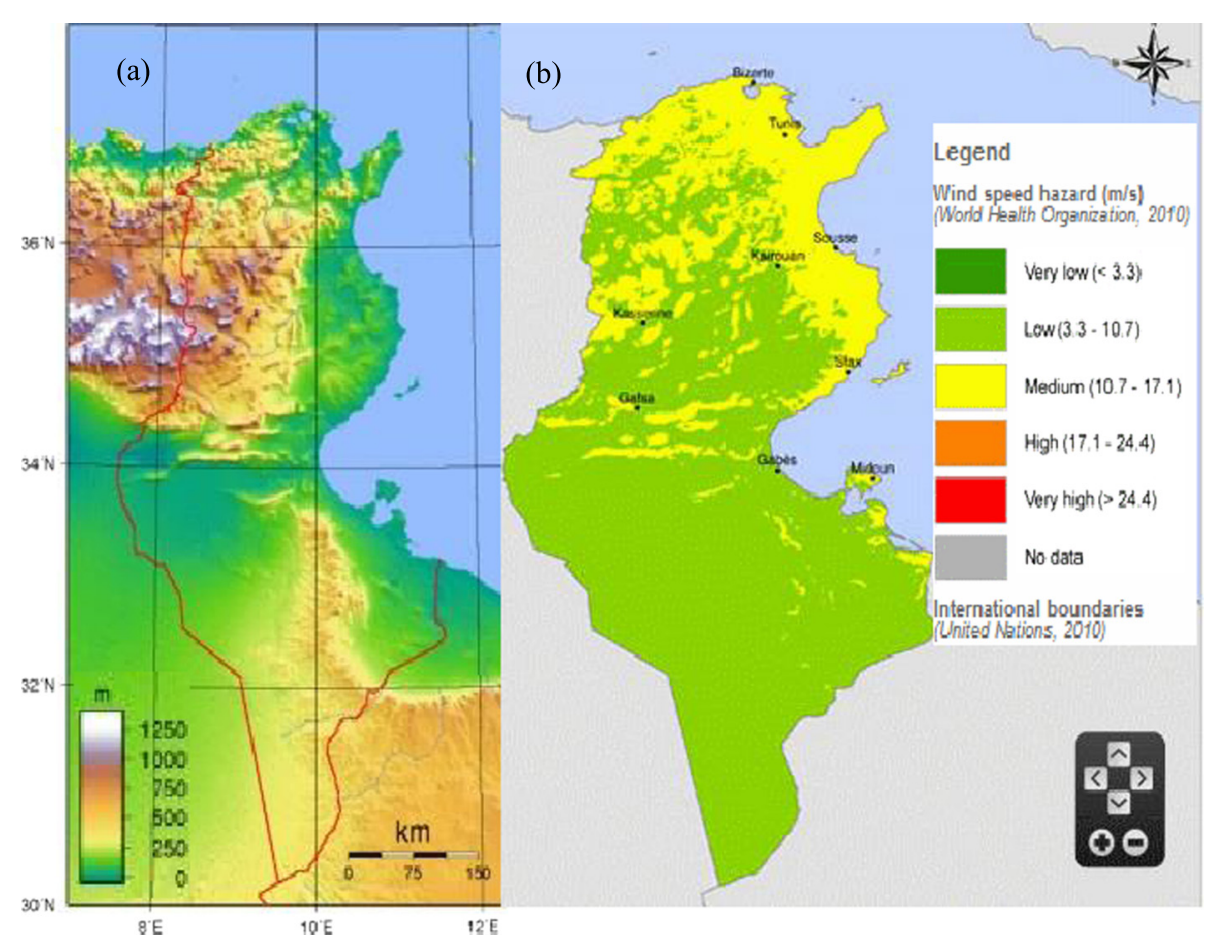

Fig. 1. Tunisia map: (a) Topographic map and (b) map of average wind speed [3].

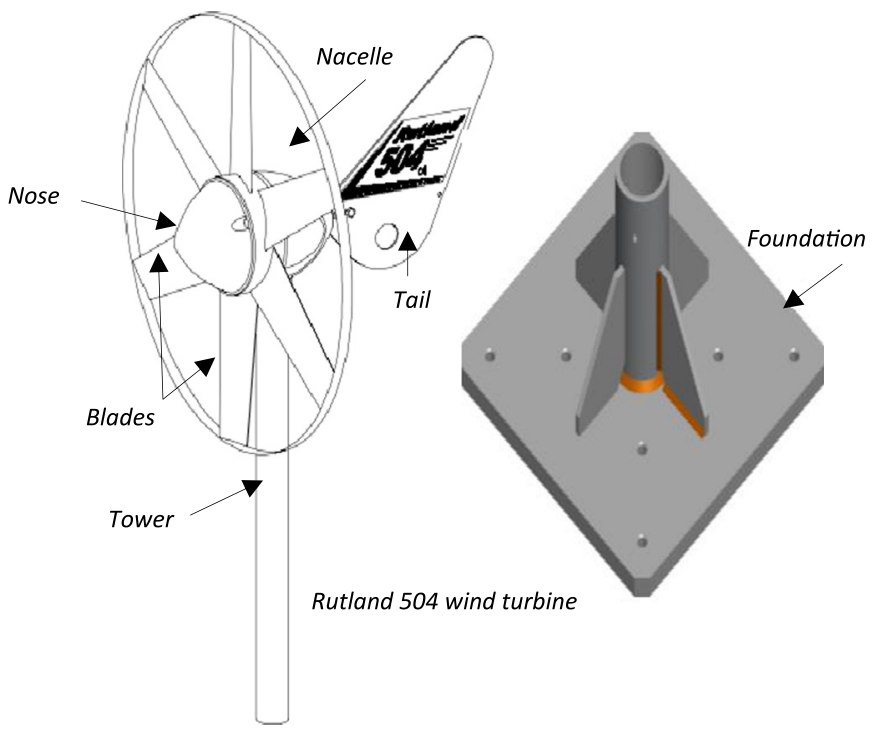

Fig. 2. The Rutland 504 wind turbine system: turbine/tower geometry and foundation.

volumes methods for analyzing the static and dynamic behaviors of different engineering structures $[8,9]$. Nevertheless, designing more and more complex wind turbines, led engineers to improve parallely their experimental techniques in order to allow the accurate calculation of static and dynamic parameters [10]. Experimental modal analysis affords the reliable information required to validate the numerical methods-based models [11]. Furthermore, numerical analysis affords reliable information about the frequency range selection, the localities and sensitivity of used sensors [12].
The experimental modal analysis can be achieved using an impulse hammer to stimulate the structure vibration modes. Impulse hammer has the quality of providing a large band of inputs and outputs. However, this tool can be limited by the energy lack to stimulate some important vibration modes besides the limits of size of the structure. Thus, electrodynamic shakers can present a good solution as they provide a large variety of powerful input signals (sinusoidal, chock, random, etc.) [13]. These shakers are able to stimulate turbine structures with a high frequency resolution.

This paper is structured as follows: In the second section, the wind turbine vibrations are numerically analyzed with ANSYS. Then, an experimental validation, by TREVISE vibration platform, of the numerical model is described in the third section basing on modal testing. Hence, hammer-based excitation is first realized. Then electrodynamic shaker based-modal testing is then achieved. A comparison between the two tools results are then presented. Finally, conclusions are presented.

\section{Wind turbine numerical model}

The studied wind turbine system, which is Rutland 504, is presented in Figure 2. The characteristics of the turbine components are presented in the appendix. The Rutland 504 is six-bladed turbine with a specific design. The presence of the sharp nose dome is in order to maximize the wind flow. The Long tail is added to enhance the turbine orientation to wind. The circular stabilization ring permits the protection of the blades. An adequate foundation is designed to maintain the wind turbine fixed to the soil as presented in Figure 2. 

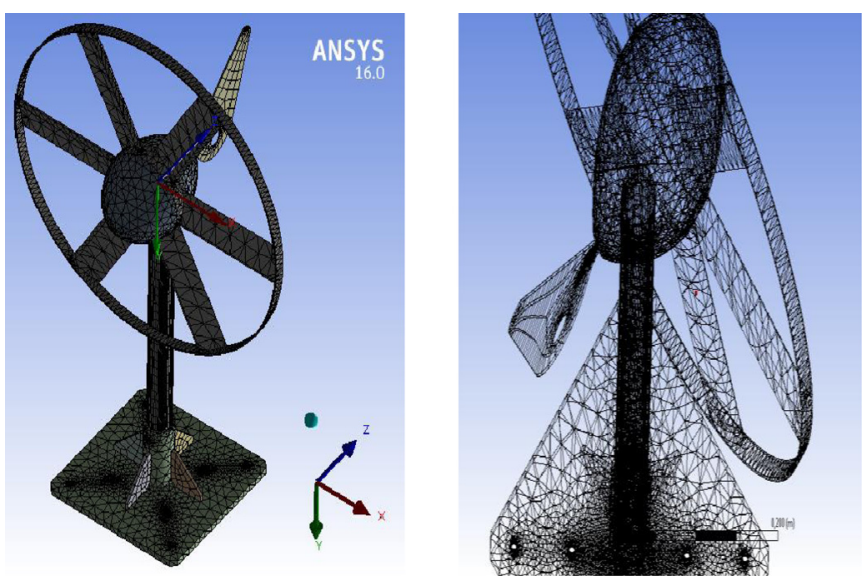

Fig. 3. 3-D ANSYS meshed model of the turbine/tower/ foundation structure with $(x, y, z)$-reference frame.

Following calibration, the geometry dimension representing the rotor blades, the tower and the foundation are used to conduct numerical simulations. The components are modeled as 3-D flexible body systems having flexible and damping properties. After designing the 3-D system geometry, materials of each component are fixed. The tower and the foundation of the turbine are constructed from steel to resist the heavy weight of the nacelle and endure the forces exercised by the rotating blade. The blades, the nose, the tail and the nacelle are constructed from complex materials with a good UV stability and durability. The boundary conditions of the structure are then well established. The foundation of the wind turbine is forced to be fixed to the ground in order to block the tower. The $x, y$ and $z$ axes are defined, as flapwise ( $x$-axis), edgewise ( $z$-axis) and spanwise ( $y$-axis) directions.

An adequate meshing is performed to present the structure areas where the deformations and vibrations are significant as presented in Figure 3. The meshed model is composed of 53361 linear elements and 111252 nodes. It is a small sieving mesh with an edge length of $1.45 \mathrm{~mm}$ and a high smooth factor. It is noted that a powerful pc processor is required in order to handle the large deflections of the structure and its number of degrees of freedom.

Free-vibration analysis is performed after fixing the suitable boundary condition in order to evaluate the model modal parameters. The general equation of the structure motion is solved to determine the natural frequencies and mode shapes of the turbine/tower/foundation structure.

The first calculated natural frequencies of the turbine model are examined as presented in Figure 4. The first calculated natural frequency is $17.2 \mathrm{~Hz}$ as presented in Figure 4a. This high value is expected for such small structures like the tested micro-turbine. Then, the natural frequency increases to $28.9 \mathrm{~Hz}$. For the $3 \mathrm{rd}$ mode shape, the frequency reaches $39.2 \mathrm{~Hz}$. The 4 th mode is characterized by a natural frequency of $47.7 \mathrm{~Hz}$. Calculating the structure displacements allow to find out the areas where the maximum stress concentration occur. The maximum displacement is presented by a red color in the figure. A comparison between the total displacement distributions corresponding to the first four modes is also presented in Figure $4 \mathrm{a}-\mathrm{d}$. As it can be seen, the tail, then the six blades tips and then the outer ring are the components most vibrating awarding both flapwise and edgewise directions.

\section{Wind turbine experimental modal analysis}

Experimental modal analysis is still the trustiest method identifying the structural parameters. The method allows validating designed numerical models [14]. After exciting the turbine/tower/foundation by means of a known force $\{F(t)\}$, the vibration response $\{u(t)\}$ over the structure is evaluated. Then, Fast Fourier Transform (FFT) analyzer generated by the data acquisition system, is adapted to calculate the excitation spectrum vector $\{F(j \omega)\}$ and the response spectrum vector $\{u(j \omega)\}$. Then, frequency response functions (FRFs) are calculated. These functions contain all necessary information to evaluate the modal parameters of structures. The following expression presents the linear response of the turbine under test to the excitation force

$$
\{u(j \omega)\}=[R(j \omega)]\{F(j \omega)\},
$$

where $[R(j \omega)]$ presents the receptance spectrum matrix of the FRFs. The other characteristics of FRF matrices can be identified by

$$
\begin{aligned}
& {[M(j \omega)]=\frac{\{\dot{u}(j \omega)\}}{\{F(j \omega)\}},} \\
& {[A(j \omega)]=\frac{\{\ddot{u}(j \omega)\}}{\{F(j \omega)\}},}
\end{aligned}
$$

where $[M(j \omega)]$ is the mobility matrix defined as the matrix of mobility of the FRFs. $[A(j \omega)]$ is the accelerance matrix defined as the matrix of accelerance of the FRFs.

The Hermetian matrix of equation (1) is defined by

$$
\{u(j \omega)\}^{H}=[R(j \omega)]^{H}\{F(j \omega)\}^{H} .
$$

Multiplying equation (3) by its Hermetian matrix, we get

$$
\{u(j \omega)\}\{u(j \omega)\}^{H}=[R(j \omega)]\{F(j \omega)\}\{F(j \omega)\}^{H}[R(j \omega)]^{H} .
$$

Naming $\left[G_{u u}(j \omega)\right]=E\{u(j \omega)\}\{u(j \omega)\}^{H}$ the matrix of the $\left(m^{*} m\right)$ output spectral density, where $m$ is the number of outputs. $\left[G_{F F}(j \omega)\right]=E\{F(j \omega)\}\{F(j \omega)\}^{H}$ is the $\left(r^{*} r\right)$ matrix of the input spectral density, where $r$ is the number of inputs. $[R(j \omega)]$ is then the $\left(m^{*} r\right)$ FRF receptance spectrum matrix. Equation (5) turns to

$$
\left[G_{u u}(j \omega)\right]=[R(j \omega)]\left[G_{F F}(j \omega)\right][R(j \omega)]^{H} .
$$



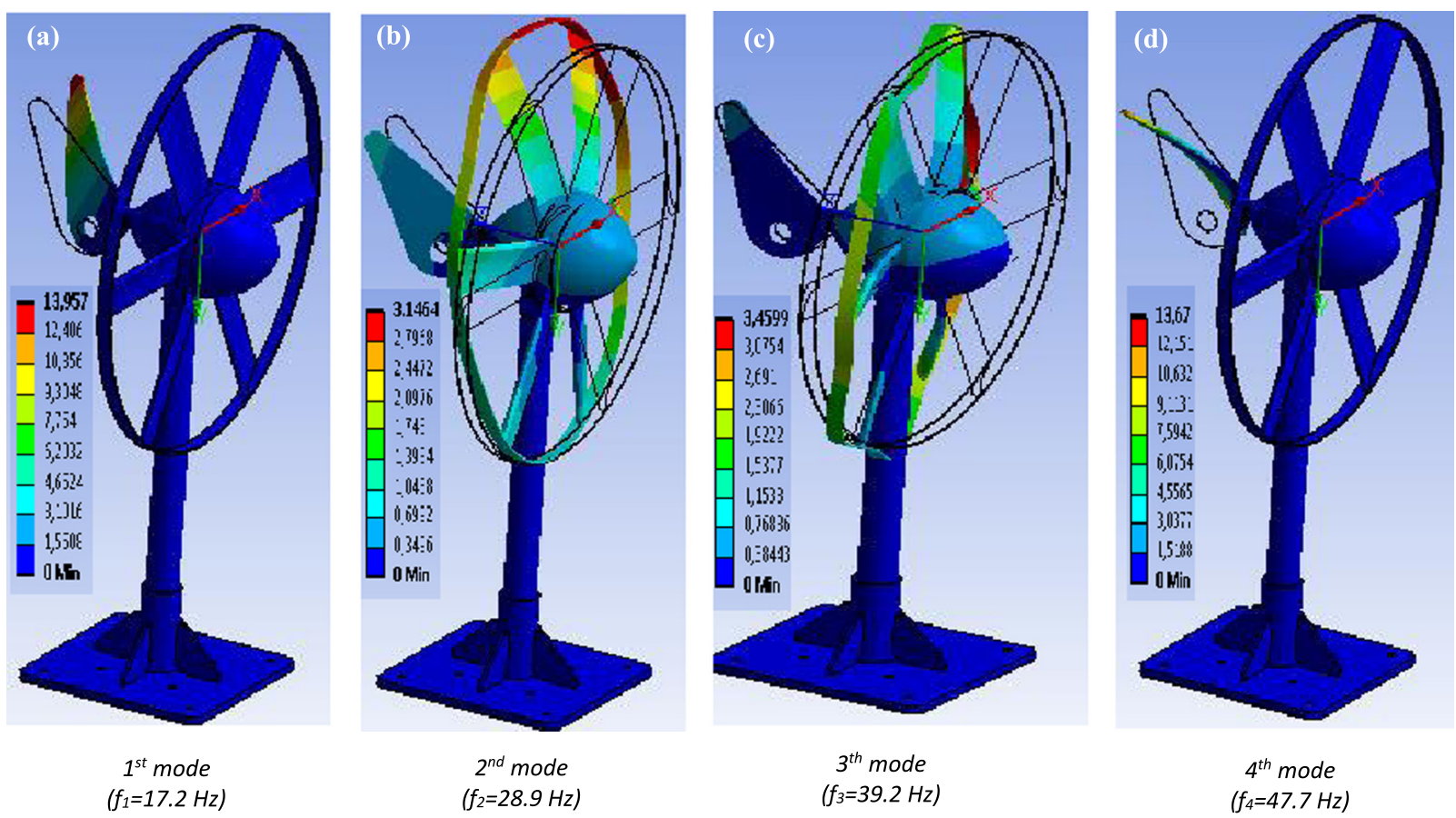

Fig. 4. Modal displacements of tested wind turbine calculated by ANSYS relative to (a) the 1st to (d) the 4th mode shapes.
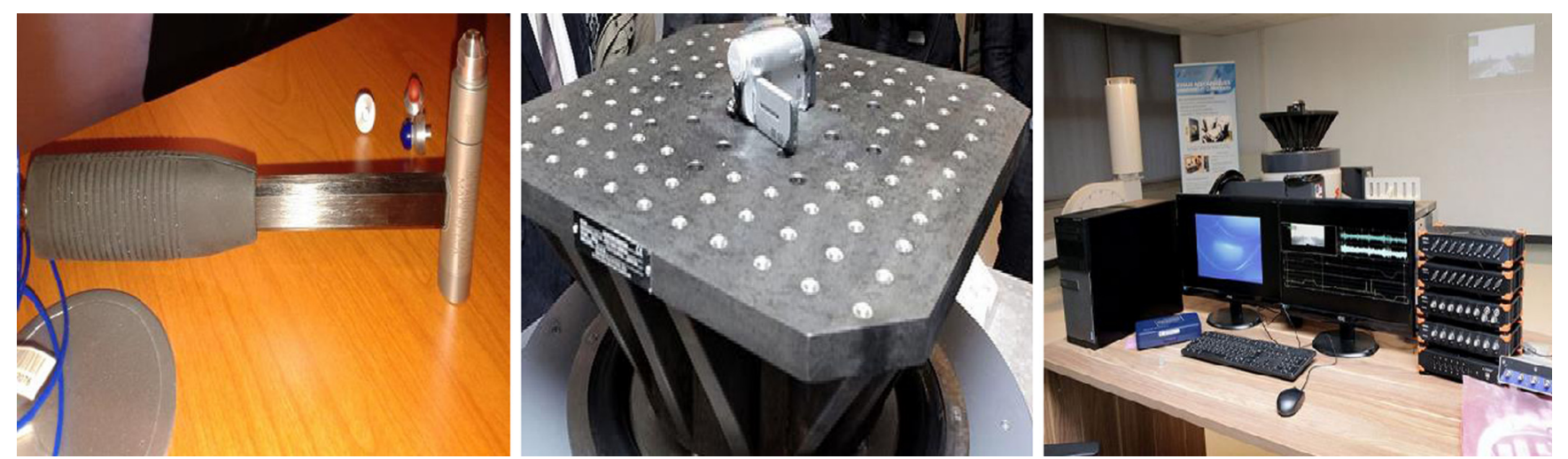

Fig. 5. Components of the vibration analysis platform TREVISE: the impact hammer, the shaker and the data acquisition package.

The frequency responses functions can be expressed by the following partial fraction

$$
[R(j \omega)]=\sum_{k=1}^{n} \frac{R_{k}}{j \omega-\lambda_{k}}+\frac{\bar{R}_{k}}{j \omega-\bar{\lambda}_{k}}
$$

where $n$ is the modes number of the tested structure. $\lambda_{k}$ and $R_{k}$ are respectively the pole and the residue of the transfer function. The expression of the residue $R_{k}$ can be defined by

$$
R_{k}=\varphi_{k} \gamma_{k}^{T},
$$

where $\gamma_{k}$ and $\varphi_{k}$ present the modal participation vector and the mode shape vector, respectively.

\subsection{Experimental setup: vibration analysis platform (TREVISE)}

The experimental modal testing have been performed in the vibration analysis platform TREVISE. The platform ensures the understanding of vibration phenomena of different embedded systems. It is constituted of a collection of instruments, including a vibrator that can accommodate items up to $500 \mathrm{~kg}$, shakers and two machines for vibroclimatic tests for vibrations endurance check under extreme temperatures $\left(-100 /+200^{\circ} \mathrm{C}\right)$. Figure 5 presents TREVISE shaker supporting objects up to $500 \mathrm{~kg}$ for weight and $100 \mathrm{~g}$ for accelerations and a calibrated impact hammer with the data acquisition package (DEWESOFT).

An accelerometer is fixed on the shaking table to measure the vibratory motion during the random, swept sine or shock test. The electromechanical muscle of the 


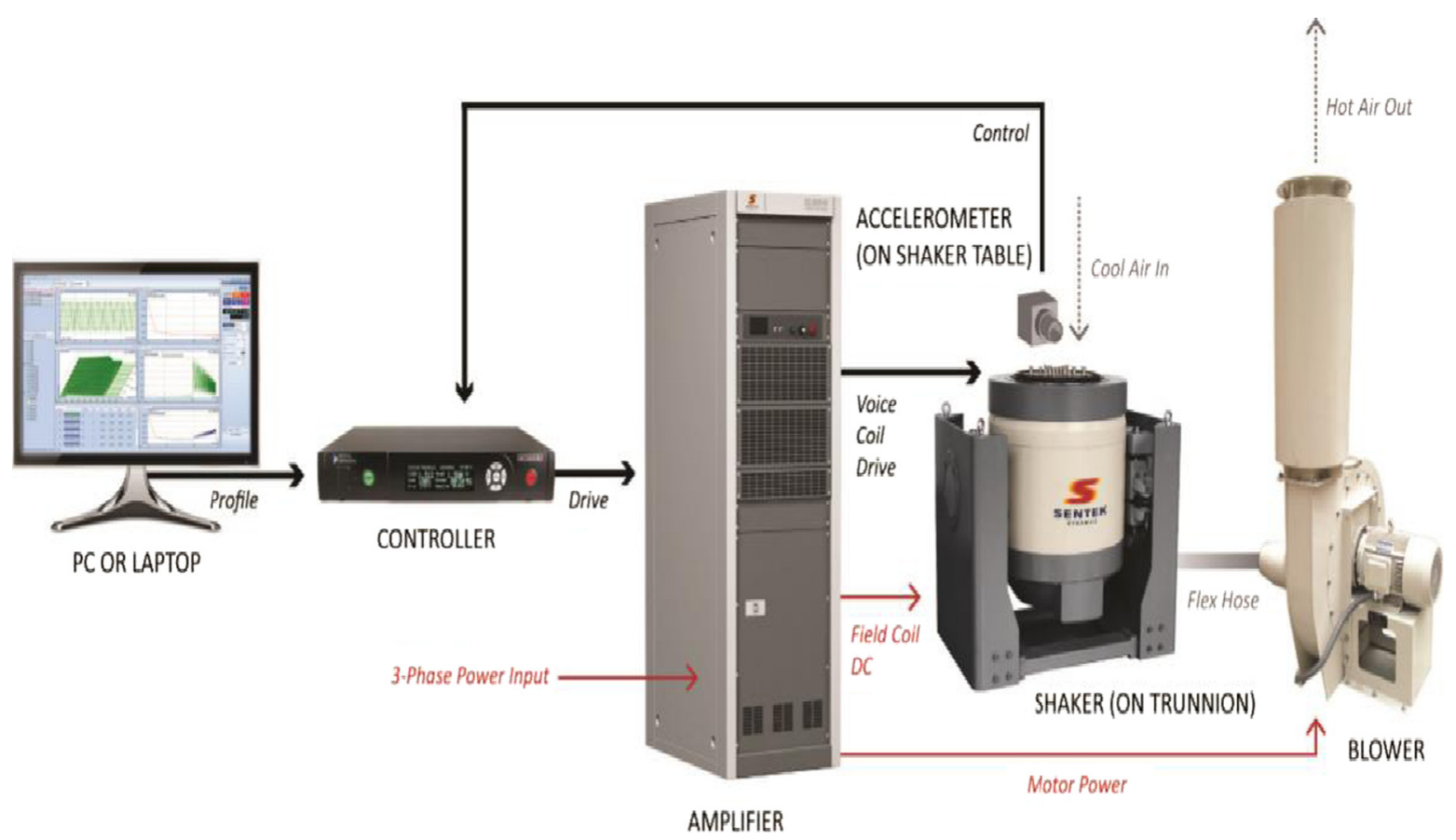

Fig. 6. Overview of the components of TREVISE vibrator shaker-based.

shaker has to provide a sufficient force to move the item under test with the desired acceleration level as presented in Figure 6. The shaker is connected to an amplifier and a controller. The controller compares continuously the measured control signal to the desired reference profile specified for the test. Then, it calculates the needed drive signal for the shaker to power the amplifier. An adequate frequency bandwidth is also required to fit in the test's specification.

Afterward, signals are stored into one file by the data acquisition package. Thus, the package transforms the physical inputs (acceleration, force, current and strain) into legible digital signals. Then, the digital signals are analyzed, filtered and post-processed. FFT analyzer is likewise used for signals spectral analysis with different frequency resolution. The forces are applied at several points along the volume structure with an adequate frequency resolution. FRF are the transfer functions in the frequency domain generated by DEWESOFT during the modal testing. They correspond to the quotient of responses to excitations. Indeed, a Mode Indicator Functions (MIF) is generated to help in determining the transfers function poles (natural frequencies) of the structure. Once the poles are calculated, the poles and residues are generated to estimate the mode shape coefficients. These coefficients are then adopted to animate the structure mode shapes.

\subsection{Wind turbine experimental modal testing}

First, an adequate steel foundation is constructed to maintain the Rutland 504 wind charger fixed to the shaker table during the modal testing. A powerful airflow simulator was taken during tests to operate the turbine at different wind speeds. The speeds are measured by a
PCE-DT62 tachometer. The tested turbine is coupled to a $12 \mathrm{~V}$ lead acid battery. The battery is protected from overcharging and damage by HRS504 regulator. An oscilloscope is placed to measure voltage and current signals generated by the turbine.

It should be noted that the mass of the accelerometers must not exceed $10 \%$ of the tested structure mass [15-17]. For our turbine/tower/foundation structure, with a total mass of $7 \mathrm{~kg}$, we are limited to fifteen piezoelectric accelerometers. These accelerometers are equipped with integrated coaxial cables of very small diameters in order to not disturb the vibratory responses of the structure. The positions and sensitivities of the used accelerometers are selected basing on the numerical model results presented in Figure 4.

\subsubsection{Hammer-based modal testing}

To evaluate the modal characteristics of the wind turbine, hammer-based testing can be exploited. This technique allows to ensure the presence of energy in the frequency range of interest able to evaluate a maximum of modes of the structure. The response of the system to an excitation can basically be represented as a sum of the contributions from all the modes of the system. The turbine basic components shapes are measured when the turbine foundation is subjected impact chock loadings following $z$-axis direction as presented in Figure 7.

Under external forces ensured by the hammer, flapwise ( $x$-axis), edgewise ( $z$-axis) and spanwise ( $y$-axis) vibrations of the turbine components were measured. Thus, fifteen PCB accelerometers with high sensitivities were attached in many locations on the tower, the nacelle and the blades of the turbine under test as presented in Figure 7 . The accelerometers are calculating the continuous accelerations 

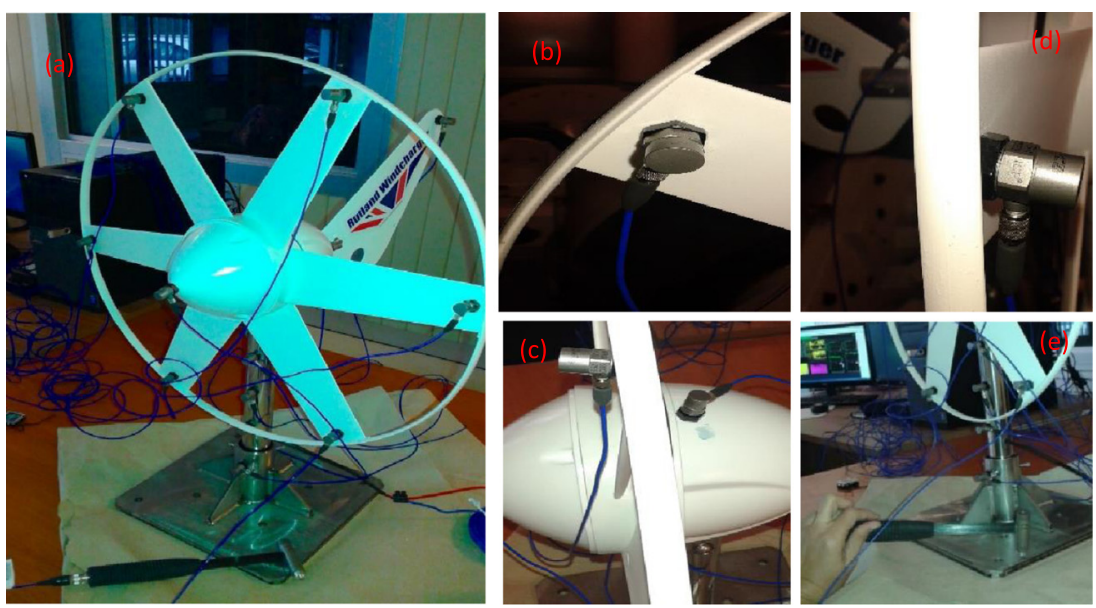

Fig. 7. (a) The turbine/tower/foundation and the test instrumentations, (b, c and d) the accelerometres placements and (e) the calibrated hammer testing.
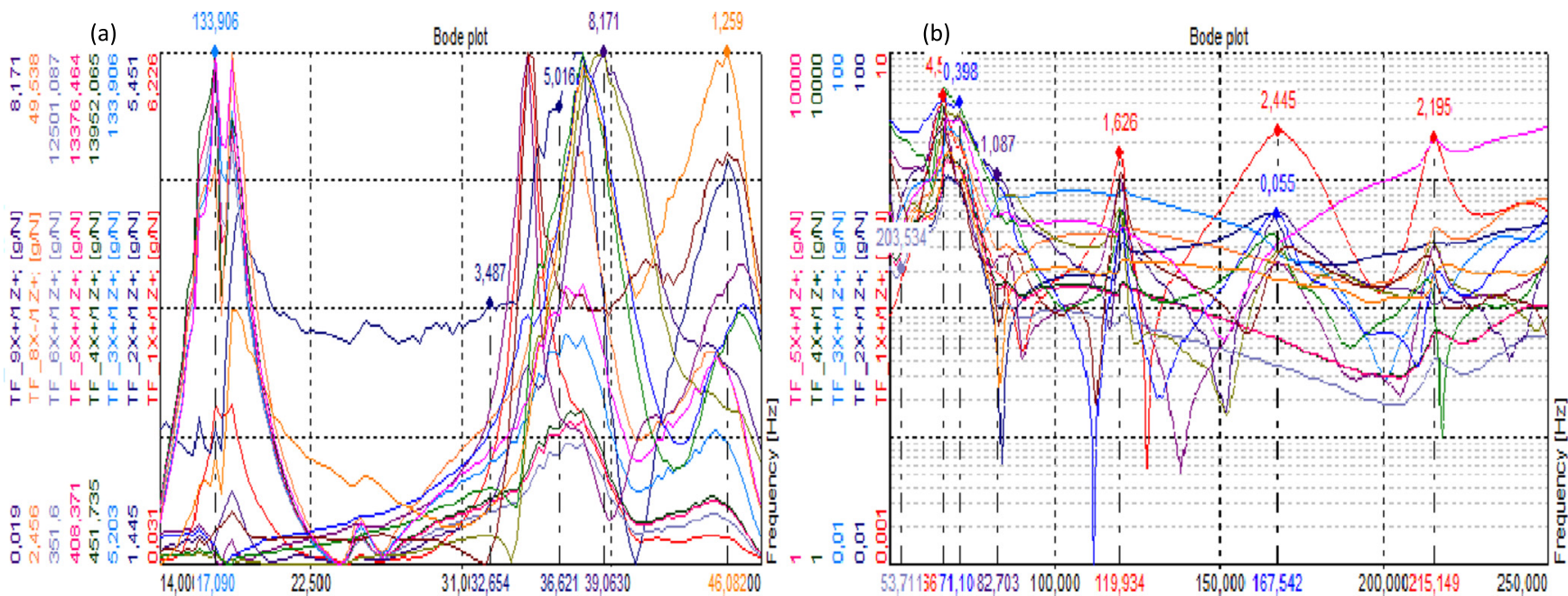

Fig. 8. Experimental measured natural frequencies in the frequency band (a) $[0-50 \mathrm{~Hz}]$ and (b) $[50-250 \mathrm{~Hz}]$.

functions in time domain. From accelerations, all other vibration variables like the velocities and the displacements can be estimated.

Figure 8 presents the calculated FRF spectra. Then, modal parameters are extracted (natural frequencies and mode shapes) basing on the Peak Picking (PP) method. The PP method is widely utilized to evaluate the modal parameters of complex structures. It calculates the modal parameters of complex mechanical structures from outputs and inputs signal processing calculations. The method principle consists on gathering the common peaks of FRF spectra densities in an increasing order based on the stability diagram analysis. However, not all the detected peaks correspond to mode shapes. Thus, selected peaks are then checked by the MIF generated by DEWESOFT. The first verified peak is relative to the lowest mode shape of the wind turbine that is the first mode.

For the first frequency band $[0-50 \mathrm{~Hz}]$ presented in Figure 8a, the wind turbine is characterized by only four experimental measured modes out of eight ones. The experimental measured natural frequencies start from
17.1 Hz for the 1 st mode shape. The 2 nd natural frequency saved is $32.6 \mathrm{~Hz}$ relatively to the second mode shape. The natural frequencies carry on increasing to reach $36.6 \mathrm{~Hz}$ at the 4 th mode and $39.1 \mathrm{~Hz}$ at the 5 th mode. The measured natural frequency value at the 6 th mode is $71.1 \mathrm{~Hz}$ as presented in Figure 8b. The measured natural frequencies carry on increasing to reach $167.4 \mathrm{~Hz}$ at the 13 th mode.

\subsubsection{Shaker-based modal testing}

Experimental mode shapes can be estimated from measured spectral densities using the shaker presented in Figure 9. To ensure the presence of suffisiant energy in the frequency range of interest able to evaluate the maximum of modes, sine-sweep excitation is selected to excite the turbine foundation. This exitation force $\{F(\mathrm{t})\}$ is ensured by the electrodynamic shaker. Then, the spectrum matrix $\left[G_{u u}(j \omega)\right]$ is evaluated. Many FRF spectra densities measured by various accelerometers on different locations of the turbine components are taken into account to improve the results. 
Table 1. Comparison between numerical and shaker-based EMA and hammer-based EMA results.

\begin{tabular}{|c|c|c|c|c|c|}
\hline Mode & ANSYS frequency $(\mathrm{Hz})$ & $\begin{array}{l}\text { Shaker-based EMA } \\
\text { frequency }(\mathrm{Hz})\end{array}$ & Relative error (\%) & $\begin{array}{l}\text { Hammer-based EMA } \\
\text { frequency }(\mathrm{Hz})\end{array}$ & Relative error (\%) \\
\hline 1 & 17.2 & 19.5 & 11.9 & 17.1 & 0.6 \\
\hline 3 & 39.2 & 39.1 & 0.4 & 39.1 & 0.4 \\
\hline 4 & 47.7 & 48.8 & 2.3 & 46.9 & 3.4 \\
\hline 7 & 82.5 & 87.8 & 5.9 & 82.8 & 0.1 \\
\hline 8 & 100.4 & 97.6 & 2.9 & missed & - \\
\hline 9 & 112.4 & 107.4 & 5.5 & missed & - \\
\hline 10 & 114.8 & 117.2 & 4.0 & 119.9 & 4.3 \\
\hline 11 & 130.0 & 127.0 & 2.3 & missed & - \\
\hline 15 & 187.3 & 185.5 & 0.9 & missed & - \\
\hline
\end{tabular}

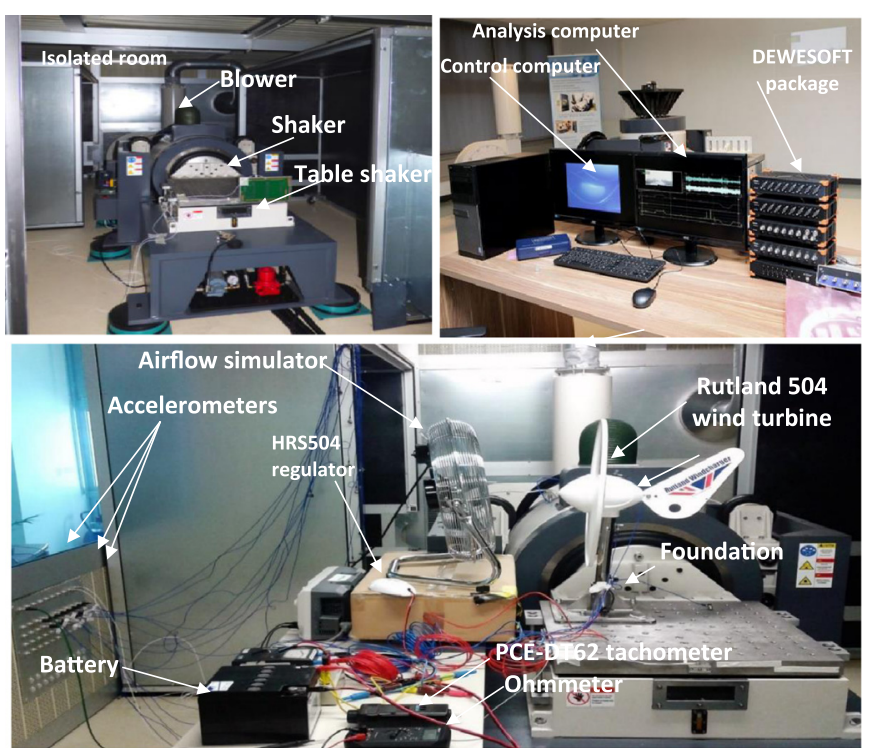

Fig. 9. Experimental setup of TREVISE platform with the test instrumentations: Rutland 504 wind turbine testing.

It is remarked during tests that the appearance of modes depends on the power and the form of the excitation force because a sufficient energy has to be injected to the structure to show up the maximum of modes. It is observed that the measured natural frequencies are relatively stable. Their values do not depend on the excitation signal form and frequency. It is also noted that random and choc excitations do not stimulate all the structure modes. Only some natural frequencies in the frequency ranges of interest can be identified. However, swept-sine excitations are the best exciting signals able to stimulate a maximum of modes and natural frequencies. Figure 10 presents the measured FRF accelerance spectra densities in the frequency range
[0-200 Hz]. The first modes of the wind turbine are examined and the modal parameters are extracted (natural frequencies and mode shapes) basing on the PP method. For the first frequency band $[0-100 \mathrm{~Hz}]$ presented in Figure 10a, the experimental measured natural frequencies start from $19.5 \mathrm{~Hz}$ for the 1 st mode shape. The 2 nd natural frequency saved is $29.2 \mathrm{~Hz}$ relatively to the second mode shape. The natural frequencies carry on increasing to reach $39.1 \mathrm{~Hz}$ relative to the $3 \mathrm{rd}$ mode and $48.9 \mathrm{~Hz}$ relative to the 4 th mode. The measured natural frequency value relative to the 6th mode is $78.1 \mathrm{~Hz}$. The measured natural frequencies carry on increasing to reach $185.5 \mathrm{~Hz}$ relatively to the 15 th mode.

\subsubsection{Discussion and numerical model validation}

The lowest natural frequencies of the wind turbine determined by both hammer and shaker-based method are compared to those calculated by the numerical approach using ANSYS. It has to be noted that both the numerical and experimental uncertainties are quantified. Hence, the experimental natural frequencies values are used to modify the parameters of the numerical model until having numerical natural frequencies close to the experimental values.

Table 1 presents the experimental and numerical computation final results in the frequency range [0-200 Hz]. The relative error (Relative errors $(\%)=\mid($ ANSYS-EMA $)||$ ANSYS [\%]) is then calculated for each mode for hammerbased EMA and shaker-based EMA methods. The calculated relative errors are small. In fact, the maximum error does not exceed $12 \%$. These results assert an excellent correlation between hammer- and shaker-based experimental measurements and numerical calculations.

For hammer-based EMA, the 2nd mode was not picked although the 2 nd and the 3 rd numerical natural frequencies have not close values $(28.9 \mathrm{~Hz}$ and $39.2 \mathrm{~Hz})$. The fifth mode 

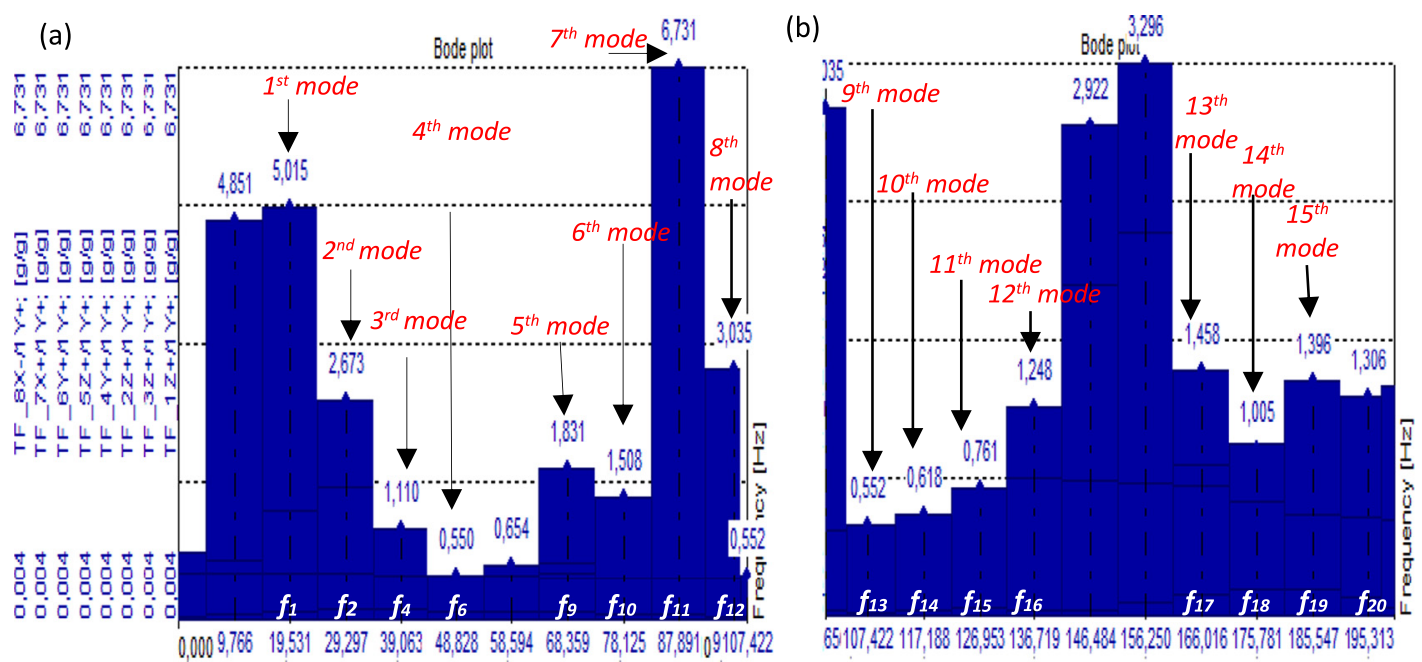

Fig. 10. Experimental measured natural frequencies in the frequency band (a) $[0-100 \mathrm{~Hz}]$ and (b) [100-200 Hz].

Table 2. Characteristics of the wind turbine.

\begin{tabular}{ll}
\hline Nominal power & $60 \mathrm{~W}$ \\
Radius, R & $0.26 \mathrm{~m}$ \\
Tower attitude & $0.35 \mathrm{~m}$ \\
Number of blades & 6 \\
\hline
\end{tabular}

was also missed. From $100 \mathrm{~Hz}$ a lot of mode are not extracted. For the studied frequency band, only 7 experimental measured modes out of 15 ones could be extracted by the hammer-based modal testing. However, for the shaker-based EMA, all the modes have been identified. These good correlations between numerical and experimental results can validate the FVM model.

\section{Conclusion}

A numerical model of a full-scale complex designed micro wind turbine was designed by the finite volumes method. Experimental results from both shaker and hammer-based modal testing of the turbine coupled to its tower/ foundation were presented. The vibrations were measured in several positions of the structure using piezoelectric accelerometers connected to the dynamic data acquisition system. The model was finally up dated by reducing the errors between the natural frequencies measured by the experimental tests and the ones calculated by the numerical method. Finally, an excellent correlation between experimental and numerical technique allows the validation of the finite volumes-based model. The validated model will be used in a future work for modal parameters-based damage detection approach for the tested turbine under faulty conditions.

It is found that shaker-based EMA technique is able to assess all the calculated modes of the turbine using sinusoidal excitation. However, the hammer-based EMA
Table 3. Characteristics of the shaker (M4040A) of TREVISE platform.

\begin{tabular}{ll}
\hline Sine force peak, $\mathrm{kN}(\mathrm{lbf})$ & $40(8800)$ \\
Random force, rmskN (lbf) & $40(8800)$ \\
Shock force $(6 \mathrm{~ms}), \mathrm{kN}(\mathrm{lbf})$ & $80(17.600)$ \\
Frequency range (Hz) & $5-2200$ \\
Continuous displacement, mm (in) & $51(2.0)$ \\
Shock displacement, mm (in) & $51(2.0)$ \\
Max velocity, m/s (in/s) & $2.0(78.7)$ \\
Max acceleration sine peak, m/s ${ }^{2}(\mathrm{~g})$ & $980(100)$ \\
Armature diameter, mm (in) & $400(15.8)$ \\
Effective armature mass, kg (lb) & $40(88.0)$ \\
Max static payload, kg (lb) & $500(1100)$ \\
\hline
\end{tabular}

did not ensure enough energy to excite all the wind turbine modes. The work proves that the tail, the blades tips and the ring are the most sensitive parts to vibrate of modern micro-turbine. It is due to their particular geometries and materials. Based on this structural validated model, dynamic analyses under multiple shock and seismic events can now be investigated studying the temporal and frequency variation of the modal parameters of the components of the turbine and estimating their fatigue damage. Operational modal analysis will also be realized in a future work in order to extract the wind turbine modal parameters under operation conditions.

Acknowledgements. This work was realized using TREVISE, a mechatronic platform conjointly financed by the FEDER fund, the Ile-de-France region, the Val-d'Oise department and the Urban Community of Cergy-Pontoise, which are gratefully acknowledged. 


\section{Appendices Appendix: Wind turbine characteristics}

The tested wind-turbine is a Rutland 504 micro wind turbine. The tower of the turbine is constructed from steel. The blades and the nacelle are constructed from complex materials. These materials are not published online. Table 2 presents the dimensions of the designed micro-wind turbine.

The characterstics of the used electrodynamic shaker of TREVISE platform are presented in Table 3.

\section{References}

[1] L. Baringo, A.J. Conejo, Strategic wind power investment, IEEE Trans. Power Syst. 29 (2014) 1250-1260

[2] T. Sellami, Contribution à l'usage de l'analyse vibratoire comme outil de monitoring et de diagnostic d'avaries pour les machines électriques tournantes, thèse, Unversité de parisSeine/Cergy Pontoise et Université de Monastir

[3] www.Mappi.net-www.Cartograf.fr@2009-2016 OnMyWeb Production, open source website

[4] T. Maatallah, N. Ghodhbane, S.B. Nasrallah, Assessment viability for hybrid energy system (PV/wind/diesel) with storage in the northernmost city in Africa, Bizerte, Tunisia, Renew. Sustain. Energy Rev. 59 (2016) 1639-1652

[5] J. Sopanen, V. Ruuskanen, J. Nerg, J. Pyrhonen, Dynamic torque analysis of a wind turbine drive train including a direct-driven permanent-magnet generator, IEEE Trans. Ind. Electron. 58 (2011) 3859-3867

[6] M.S. Allen, M.W. Sracic, S. Chauhan, M.H. Hansen, Outputonly modal analysis of linear time-periodic systems with application to wind turbine simulation data, Mech. Syst. Signal Process. 25 (2011) 1174-1191

[7] M.H. Hansen, K. Thomsen, P. Fuglsang, T. Knudsen, Two methods for estimating aeroelastic damping of operational wind turbine modes from experiments, Wind Energy 9 (2006) 179-191
[8] K. Takizawa, B. Henicke, D. Montes, T.E. Tezduyar, M.C. Hsu, Y. Bazilevs, Numerical-performance studies for the stabilized space - time computation of wind-turbine rotor aerodynamics, Comput. Mech. 48 (2011) 647-657

[9] J.M. O'Brien, T.M. Young, D.C. O'Mahoney, P.C. Griffin, Horizontal axis wind turbine research: a review of commercial CFD, FE codes and experimental practices, Prog. Aerosp. Sci. 92 (2017) 1-24

[10] M.H. Hansen, Aeroelastic stability analysis of wind turbines using an eigenvalue approach, Wind Energy 7 (2004) 133-143

[11] H. Hirahara, M.Z. Hossain, M. Kawahashi, Y. Nonomura, Testing basic performance of a very small wind turbine designed for multi-purposes, Renew. Energy 30 (2005) 1279 1297

[12] M. Luczak, S. Manzato, B. Peeters, K. Branner, P. Berring, M. Kahsin, Updating finite element model of a wind turbine blade section using experimental modal analysis results, Shock Vib. (2014)

[13] J.P. Baqersad, P. Poozesh, C. Niezrecki, P. Avitabile, Comparison of modal parameters extracted using MIMO, SIMO, and impact hammer tests on a three-bladed wind turbine, in: Topics in Modal Analysis II, Springer, Cham, 8, 2014, pp. $185-197$

[14] A. Iliopoulos, R. Shirzadeh, W. Weijtjens, P. Guillaume, D. Van Hemelrijck, C. Devriendt, A modal decomposition and expansion approach for prediction of dynamic responses on a monopile offshore wind turbine using a limited number of vibration sensors, Mech. Syst. Signal Process. 68 (2016) 84-104

[15] J. Pacheco, G. Oliveira, F. Magalhães, A. Cunha, E. Caetano, Wind turbine vibration based SHM system: influence of the sensors layout and noise, Procedia Eng. 199 (2017) 2160-2216

[16] T. Sellami, H. Berriri, A.M. Darcherif, S. Jelassi, M.F. Mimouni, Modal and harmonic analysis of three-dimensional wind turbine models, Wind Eng. 40 (2016) 518-527

[17] T. Sellami, H. Berriri, S. Jelassi, A.M. Darcherif, M.F. Mimouni, Short-circuit fault tolerant control of a wind turbine driven induction generator based on sliding mode observers, Energies 10 (2017) 1611

Cite this article as: T. Sellami, S. Jelassi, A.M. Darcherif, H. Berriri, M.F. Mimouni, Experimental validation of a numerical 3-D finite model applied to wind turbines design under vibration constraints: TREVISE platform, Mechanics \& Industry 18, 806 (2017) 